\title{
Local Navigation Strategies for a Team of Robots
}

\author{
Antonio Sgorbissa \\ Laboratorium \\ DIST - University of Genova \\ antonio.sgorbissa@unige.it
}

\author{
Ronald C. Arkin \\ Mobile Robot Laboratory \\ College Of Computing - GaTech \\ arkin@cc.gatech.edu
}

\begin{abstract}
Whenever a mobile robot has to deal with an environment that is totally or partially unknown or dynamically changing, local navigation strategies are very important for the robot to successfully achieve its goals. Unfortunately, local navigation algorithms that have been proposed in the literature offer poor performance (or even fail) whenever the geometry of the free space in which the robot is requested to operate increases its complexity. In this paper, we deal with a team composed of many robots, and we show how robots navigating within an unknown environment with local communication capabilities (only line-of-sight communication is allowed) can cooperate by helping each other to achieve their own goals.
\end{abstract}

Keywords-- Navigation algorithms, multi-robot exploration, multi-robot cooperation.

\section{INTRODUCTION}

Whenever a mobile robot has to deal with an environment that is totally or partially unknown or dynamically changing, local navigation strategies are very important for the robot to successfully achieve its goals, whether it is requested to accomplish a complex mission or simply to survive in the environment. By local navigation strategies we mean algorithms that return, at each computation step, the best direction for the robot to move on the basis of its current goal and the current perception of its surroundings.

Even if in many cases the robot has an approximate knowledge of the environment in which it is requested to operate and therefore hybrid solutions deliberative/reactive are possible [1][2], local strategies are important for the robot to react in real-time to unforeseen situations; e.g., in the case of Service Robotics applications [3][4], where robots are requested to execute repetitive tasks (e.g., carrying objects, escorting people) within a populated building such as a hospital, or a museum. Moreover, whenever the environment is totally unknown and the robot can rely only on its own perceptions to achieve its goals, the availability of effective, local strategies for exploring the environment becomes even more important in determining the successful accomplishment of a given mission. Consider, for example, high risk military applications where robots are deployed and requested to explore unknown buildings (possibly occupied by hostile robots/individuals) in order to search and report the existence of any hazardous 
material or to communicate to a remote station other kinds of information which will reduce the risk of losing human lives [5]. In the following, we will take this as our reference scenario.

Unfortunately, local navigation algorithms that have been proposed in literature offer poor performance (or even fail) whenever the geometry of the free space in which the robot is requested to operate increases its complexity: artificial potential field based approaches have the tendency to lead the robot into local minima [6]; search algorithms [7] may require a long time for the robot to find a path to its goal, and are therefore inefficient whenever the time spent in exploring the environment is a factor that needs to be minimized. However, in many applications related to autonomous exploration, the use of a team of robots for maximizing the coverage of a given area is foreseen (as is the case of our high risk reference scenario). Thus, it is straightforward to try to improve the performance of each individual robot (which is given a sequence of navigation tasks and a local navigation algorithm with poor performance) by allowing teammates to cooperate by helping each other to achieve their own goals.

In [8] a formal comparison between the use of explicit and implicit communication is presented, focusing on the improvement of performances of the whole system when more robots are allowed to share with each other their own goals or their own internal state. In our system we allow the use of explicit communication between robots; however we assume that the robots are allowed to communicate only when they are in sight of each other (that is, broadcasting information to all robots throughout the building is not allowed). Limiting the system to line-of-sight communication is a natural consequence of the particular scenario we have chosen; in fact we want the robots to be able to operate in potentially hostile settings where standard radio communication may be impossible. However, as it will be shown in the following, this work does not focus on defining strategies for keeping robots in sight of each other during multi-robot exploration and coverage of the environment. Instead, we consider line-of-sight as a constraint of the problem. This line-of-sight constraint posed for communication seems to fit well with the local/reactive approach to the navigation problem: since the robots rely only on 'local knowledge' of the environment, they are assumed to allow only 'local communication'.

For example, in our reference scenario, we could imagine a situation as depicted in Figure 1: $\mathrm{M}$ robots are deployed in an unknown (or partially known) building with the purpose of exploring it and reporting to a base station what they have discovered. A possible strategy could be the following: one robot is given the task of patrolling the corridor, maintaining line-of-sight with the base station at the entrance of the building (everything must be reported 
to or through the base station) while watching for potential enemies, while other robots explore the rooms on both sides of the corridor. In the following, we will show how the robots can help each other while accomplishing their respective tasks.

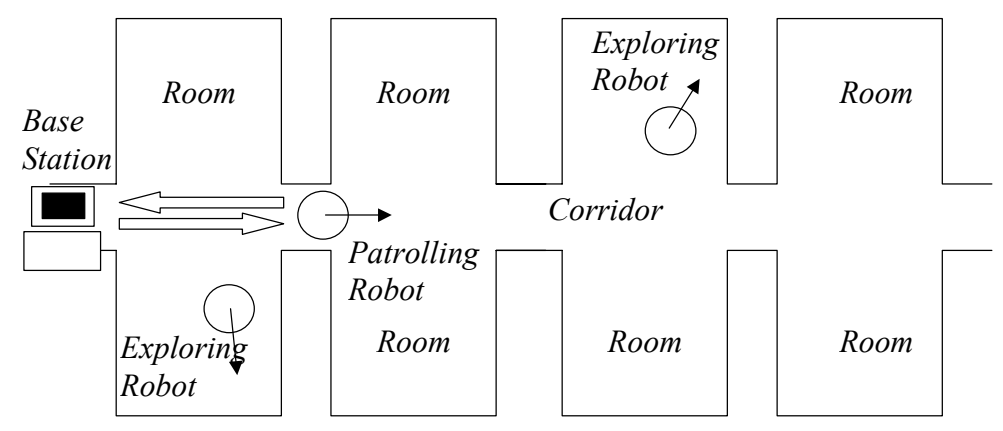

Figure 1. One robot is given the task of patrolling the corridor; other robots explore the rooms on both sides of the corridor.

\section{THE NAVIGATION PROBLEM}

In the above scenario (autonomous navigation in an unknown, potentially hostile environment), we define a navigation problem for a robot $R_{i}$ as follows:

- $R_{i}$ is given a starting point Start $_{i}$ and a set of goals $<G_{i l ., \ldots}, G_{i N}>$ to be reached in sequence. Goals can be both spatial location and interesting objects to be found, whose location is not necessarily known to $R_{i}$. When goal positions $<G_{i l ., \ldots}, G_{i N}>$ are known, they are expressed as x, y coordinates with respect to a relative reference frame $F_{i}$ that is centered on $R_{i}$ : we call $G_{\mathrm{ik}, \mathrm{i}}$ the $\mathrm{k}^{\text {th }}$ goal position with respect to $F_{i}$. The notations $R_{i}$, Start ${ }_{i}$, and $G_{\mathrm{ik}}$ are used to indicate the corresponding quantities with respect to a hypothetical absolute reference frame common to all robots that is used for the sake of simplicity in the following explanation. This absolute reference frame is not known nor used by the robots themselves. Finally, during each interval $\left[\mathrm{t}_{\mathrm{ik}}, \mathrm{t}_{\mathrm{ik}+1}\right]$ only one goal $G_{i k}$ is significant for the robot (we say that $G_{i c u r r}=G_{i k}$ ), while the others are ignored. If at time $\mathrm{t}_{\mathrm{ik}+1} R_{i}$ reaches $G_{i k}$, we set $G_{i c u r r}=G_{i k+1}$. We say that, at time $\mathrm{t}_{\mathrm{ik}+1}, R_{i}$ reaches $G_{i k}$ if $\operatorname{dist}\left(R_{i}, G_{\mathrm{ik}}\right)<\mathcal{E}$, where $\varepsilon$ is a positive number that establishes a tolerance margin. 
- $R_{i}$ is provided with a local navigation algorithm $A$ which returns, at each computation step t, what is considered as the best choice for reaching the goal $G_{\text {icurr }}$. The algorithm fails whenever it is not able to guide the robot to its current goal $G_{\text {icurr }}$ (or to prove the non-existence of a path) in a finite time.

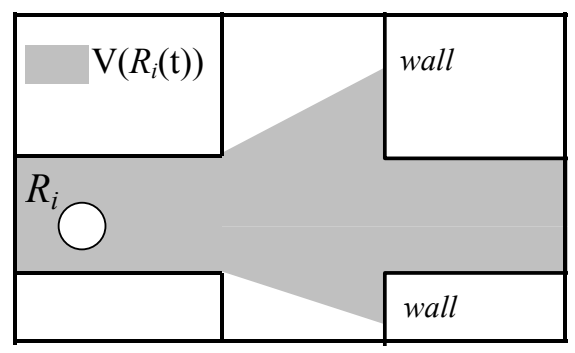

\section{Figure 2. $\mathrm{V}\left(\boldsymbol{R}_{i}(\mathrm{t})\right)$ is the visibility region of $\boldsymbol{R}_{i}(\mathrm{t})$.}

- Suppose now that the free space F is a simply connected set with polygonal boundaries. The boundary of $\mathrm{F}$ is a finite set of closed, piecewise-smooth curves with only a finite number of non-smooth points. For any $R_{i}(t) \in F$ (that is, for any configuration in the free space that $R_{i}$ can assume), let $\mathrm{V}\left(R_{i}(\mathrm{t})\right)$ denote the set of all points $V \in F$ such that the line segment that joins $R_{i}(\mathrm{t})$ and $V$ does not intersect the boundary of $\mathrm{F}$. We call $\mathrm{V}\left(R_{i}(\mathrm{t})\right)$ the visibility region (Figure 2). In the following we assume that each robot $R_{i}(\mathrm{t})$ is given omnidirectional visibility, and the corresponding visibility region $\mathrm{V}\left(R_{i}(\mathrm{t})\right)$ is contained within a circular area of bounded radius. Moreover, we say that robot $R_{i}$ can see $R_{j}$ (another robot) or $G$ (a goal) if $R_{j}$ or $G \in V\left(R_{i}\right)$. Remember that, in our definition, a goal $G$ can be either an interesting object or a location in free space.

- Whenever $R_{i}$ can see its current goal $G_{\text {icurr }}$, it starts heading towards it, by suppressing the output of algorithm $A$.

In the following we consider only the case in which the robot's goals coincide with a set of targets spatially located in the environment, whilst we ignore the case (very frequent especially in high risk, hostile environments) in which the goal coincides with a particular object (or person) to be found and identified. Thus, in our case, robots need to have some kind of approximate knowledge about the environment, since they know approximately the areas of the environment that must be explored, even if they do not know the topology of the free-space and, therefore, are incapable of planning a path. However, if we want to explore the environment to search for something, it is sufficient 
to substitute algorithm $A$ (which determines the motion strategy of the robot and has been introduced in the second point) with an algorithm for autonomous exploration (even a random-motion algorithm) without affecting the general strategy that will be proposed for multi-robot cooperation. About the fourth point, notice that $R_{i}$ chooses what he thinks to be the fastest path to the goal (this is guaranteed in a static environment) by following a straight line, no matter if $A$ proposes a different motion strategy.

\section{AGENTS COMMUNICATING THEIR OWN GOALS: COMMUNICATION IS PERCEPTION.}

Let us consider a team composed of 2 robots. We introduce a very simple strategy that allows robots to obtain the benefit of line-of-sight communication improving each robot's behavior. Suppose for the moment that $R_{l}$ is the only robot that is given a navigation problem and is therefore freely moving in the environment, while $R_{2}$ remains in a fixed position. Whenever $R_{1}$ comes in sight of $R_{2}$ (i.e. $R_{2}(t) \in V\left(R_{1}(t)\right)$ and $\left.R_{1}(t) \in V\left(R_{2}(t)\right)\right)$ :

1. Share my goal: $R_{I}$ communicates to $R_{2} \in V\left(R_{1}\right)$ its current goal $G_{\text {lcurr }}$.

2. Check visibility: If $R_{2}$ knows $G_{\text {lcurr }}$, it checks its visibility $\left(G_{1 \text { curr }} \in V\left(R_{2}(t)\right)\right)$. It then communicates the result to $R_{1}$.

3. Head to goal: If $G_{1 \text { curr }} \in V\left(R_{2}(t)\right)$ and $R_{2}(t) \in V\left(R_{1}(t)\right)$ we let $R_{I}$ infer that $G_{\text {lcurr }} \in V\left(R_{1}(t)\right)$; that is, we are introducing a transitive property in the definition of the visibility function $V(R)$. According to point 4 of the previous section, whenever $R_{1}$ can see its goal it starts heading towards it, by suppressing the output of the navigation algorithm $A$. However, since $R_{l}$ perceives its goal through $R_{2}$, it sets $G_{\text {lcurr }}=R_{2}$ and consequently heads towards $R_{2}$ until it is eventually able to directly perceive its own goal (this is guaranteed in a static environment). Obviously, this is not always possible if the robots are real and therefore they can obstruct the visibility of each other. Suppose for example that $G_{\text {Icurr }}$ is inside a room and $R_{2}$ is blocking the door: in this case, $R_{I}$ and $R_{2}$ should implement some sort of coordination strategy in order to make possible for $R_{l}$ to directly perceive its target once it has joined $R_{2}$.

Notice that the behavior of $R_{I}$ (heading towards the robot that declares to be able to see its goal) can be described by interpreting $R_{2}$ as a mirror that reflects the image of $R_{I}$ 's goal. We can imagine that $R_{l}$ heads towards the reflected image (thinking that it is the real one) until it is able to directly perceive its goal, thereby recognizing the illusion. On one side, this metaphor allows us to emphasize the parallelism between local perception/communication which 
motivates the approach: the mirror can be thought as a very simple communication device, a signal repeater that changes the direction of the visual signal: but for an external observer, what is the difference between perceiving the imagined goal reflected on the mirror and the real goal? From the other side, it allows us to easily explain the behavior of $R_{l}$ when $G_{\text {Icurr }}$ is neither directly perceived by $R_{l}$ nor by $R_{2}$, but it can be seen by a $\mathrm{j}^{\text {th }}$ robot $R_{j}$ which shares this information with $R_{j-1}$ and so on until the fact is eventually bounced to $R_{2}$ and finally to $R_{I}$ (see Figure 3 ). We can imagine robots $R_{2}$ to $R_{j}$ as reflecting mirrors that transmit to $R_{I}$ the image of its goal. $R_{I}$ starts heading towards the only goal that it can perceive (the one reflected in $R_{2}$ ), but as soon as another image is available which guarantees a shorter path to the goal (in the sense of the path traveled by light from $G_{\text {Icurr }}$ to $R_{l}$ : this concept will be clarified in the following), it changes its target and heads towards it until it is eventually able to directly perceive its own goal. In order to distinguish these concepts (visibility-through-reflection) from traditional visibility $\mathrm{V}\left(R_{i}(\mathrm{t})\right)$, we will use a different function $\mathrm{V}_{\mathrm{R}}\left(R_{i}(\mathrm{t})\right)$ that we call reflected visibility.
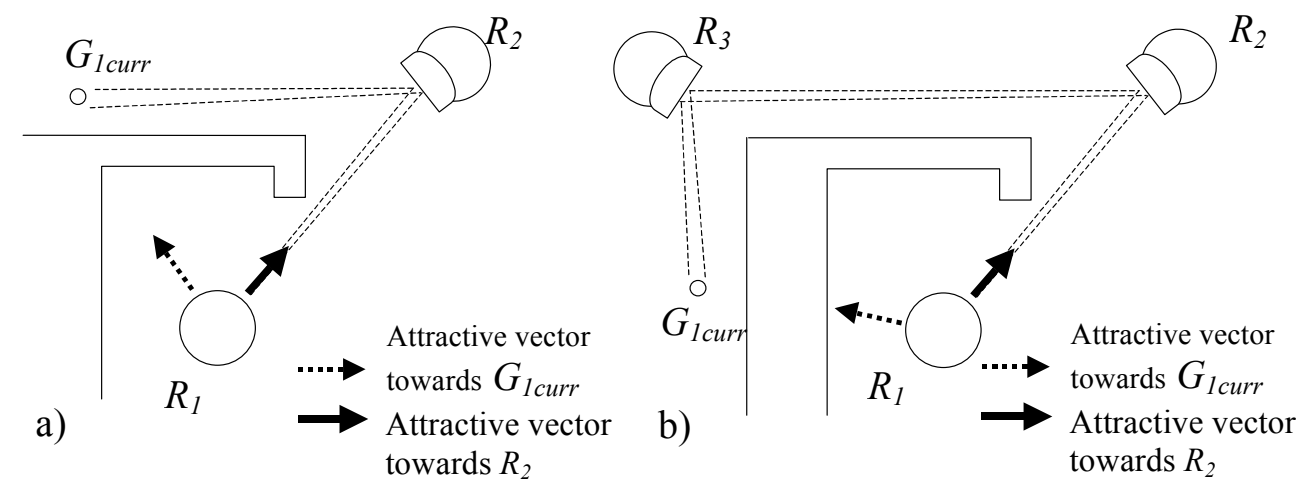

Figure 3. The behavior of $R_{1}$ when it can see its goal (a) reflected by $R_{2}$ or (b) reflected by $R_{2}$ and $R_{3}$.

In a static environment, this strategy will guarantee $R_{I}$ to finally reach its goal; however the trajectory generated is not optimum in terms of the distance that the robot has to travel. It is obviously possible to try to optimize the length of the path by considering the robots' visibility region and the geometry of the environment. However the simple behavior proposed offers another advantage: it allows the moving robot to keep its 'focus of attention' on the still robot from which it is receiving help. In many cases, this allows making visual processing faster and consequently increases the reactivity of the whole (the role played by attention in simplifying visual processing in human beings has been deeply investigated [9] [10]). 
For a generic group composed of $\mathrm{M}$ robots, the rules for the behavior of each robot can be generalized as follows (once again we assume that $R_{l}$ is the only moving robot, whilst robots $R_{2}$ to $R_{M}$ are stationary robots).

1. Share my goal: $R_{l}$ communicates to each robot $R_{i} \in V\left(R_{1}\right)$ its current goal $G_{\text {lcurr }}$.

2. Share other robots' goals: If $R_{i}$ knows $G_{\text {Icurr }}$, it communicates the information to each robot $R_{j} \in V\left(R_{i}\right)$

3. Check visibility: If $R_{i}$ knows $G_{\text {Icurr }}$ it checks its visibility $\left(G_{1 \text { curr }} \in V\left(R_{i}\right)\right.$ ). It communicates the result to each $\operatorname{robot} R_{j} \in V\left(R_{i}\right)$.

4. Reflect visibility: If $G_{1 \text { curr }} \in V_{R}\left(R_{i}(t)\right)$ and $R_{i}(t) \in V\left(R_{j}(t)\right)$ we let $R_{j}$ infer that $G_{1 \text { curr }} \in V_{R}\left(R_{j}(t)\right)$; this is now a consequence of the transitivity of $V_{R}(R)$.

5. Head to goal: Once again, if $G_{\text {lcurr }} \in V_{R}\left(R_{i}(t)\right)$ and $R_{i}(t) \in V\left(R_{1}(t)\right)$, then $R_{I}$ sets $G_{\text {Icurr }}=R_{i}$ and consequently starts heading towards $R_{i}$ until it is eventually able to perceive directly its own goal (guaranteed in a static environment). These rules will now be described in detail.

\subsection{Rules 1 and 2: share goals with other robots}

Since we ask the robots to share geometric information, we have to deal with the problem associated with the absence of a common reference frame (remember we are considering the case in which $R_{i}$ 's goals $<G_{, i 1,}, \ldots, G_{\text {iN }}>$ correspond to known Cartesian coordinates in the free space F). It is usual in many multi-robot applications to assume that the robots share some kind of global reference frame, in which all the geometrical information exchanged between robots (if any) is computed (even in reactive approaches [11]). This assumption is indeed a very strong one, since the use of a global reference frame requires robots to be aware of their position and orientation in the world with high accuracy, at least if we want the information exchanged to have the same meaning for all the robots involved in the communication process. In the following, we describe a possible solution to this problem: similar solutions have been already presented in literature: see for example [12] (while robots move, one of them remains stationary thus acting as a landmark for the teammates) and [13] (a visual pattern is used in order to retrieve distance and orientation of teammate robots through visual sensing).

It is easy to show that, because of the line-of-sight communication constraint, the global reference frame assumption is not required. More precisely, suppose that the two robots $R_{I}$ and $R_{2}$ use different relative reference 
frames $F_{1}$ and $F_{2}$. Suppose also that, at time $\mathrm{t}, R_{1}$ needs to communicate to $R_{2}$ its goal $G_{l c u r r, l}$. In order for robots to share meaningful information, $R_{2}$ needs to know the transformation matrix $T_{2 l}(\mathrm{t})$ that permits the mapping of points between $F_{1}$ and $F_{2}$, as in computing $G_{I c u r r, 2}=T_{2 l}(\mathrm{t}) G_{I c u r r, l}$. Notice that $T_{2 l}(\mathrm{t})$ is different from time to time, since robots are moving in the environment. Notice also that, for $R_{2}$ to compute $T_{2 I}(\mathrm{t})$, it should be able to recognize $R_{l}$ 's position and orientation with respect to its own reference frame $F_{2}$; however, while recognizing the position of a known object is a quite simple task (especially if the object to be recognized is designed in such a way as to be easily detected) the same is not necessarily true for its orientation. Moreover, it is well known that a wrong estimate of the rotational component of $T_{21}(\mathrm{t})$ can be a source of significant errors.

The task is much simpler if we allow $R_{1}$ and $R_{2}$ to cooperate in computing $T_{2 l}(\mathrm{t})$ (and $T_{12}(\mathrm{t})$ ). In fact, if $R_{1}$ is able to perceive $R_{2}$ 's position (x and y coordinates with respect to $F_{l}$ ) and $R_{2}$ is able to perceive $R_{l}$ 's position (x and y coordinates with respect to $F_{2}$ ), both robots are allowed to compute very accurately $T_{2 l}(\mathrm{t})$ and $T_{12}(\mathrm{t})$ by sharing information about the direction in which they see each other (see Figure 4a: $\alpha_{21}$ measures the direction in which $R_{l}$ sees $R_{2}, \alpha_{12}$ the direction in which $R_{2}$ sees $R_{l}$ ). From geometrical considerations (Figure $4 \mathrm{~b}$ ), it is straightforward to see that $R_{1}$ is allowed to compute $\theta_{21}$ (orientation of $R_{2}$ with respect to $F_{1}$ ) as $180+\alpha_{21}-\alpha_{12}$ and $R_{2}$ is allowed to compute $\theta_{12}$ (orientation of $R_{l}$ with respect to $F_{2}$ ) as $180+\alpha_{12}-\alpha_{21}$.
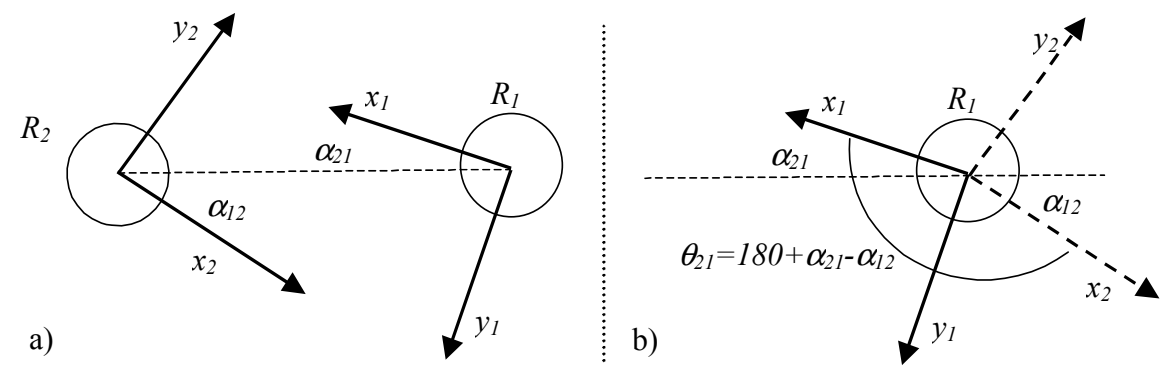

Figure 4. Robots cooperate to compute the transformation matrices $T_{21}(t)$ and $T_{12}(t)$.

Notice that, since the robots do not use a global reference frame, only $R_{l}$ is responsible for knowing with accuracy its own position with respect to the place where it needs to go. Even if $R_{2}$ is completely lost in the environment, it is still fully functional in helping $R_{l}$ to reach its target if $R_{l}$ asks it the right question (roughly speaking, we can imagine $R_{l}$ asking $R_{2}$ : can you see my goal? It should be two meters on your left...). Thus, whenever robot $R_{l}$ communicates $G_{\text {Icurr }}$ to $R_{i}$, it sends the following data: 
1. The goal position $G_{\text {Icurr }, 1}$ with respect to $F_{1}$

2. The angle $\alpha_{2 \mathrm{i}}$, corresponding to the direction in which $R_{l}$ sees $R_{i}$

3. $\mathrm{Id}_{1 \mathrm{k}}=\mathrm{k} * \mathrm{M}$, an identifier which unequivocally identifies $R_{l}$ 's $\mathrm{k}^{\text {th }}$ goal.

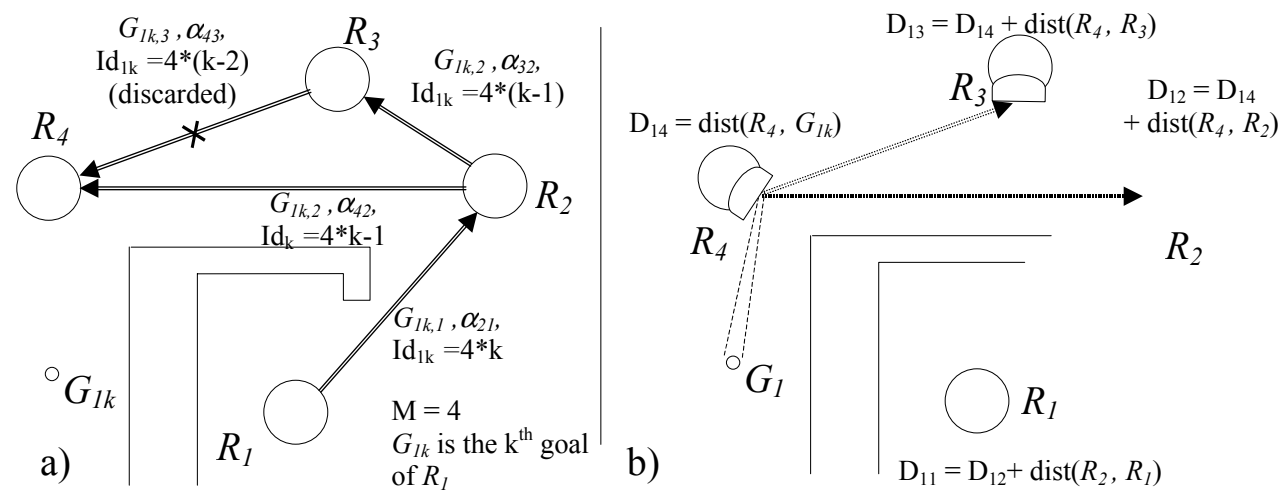

Figure 5. $R_{4}$ receives multiple reports of $G_{1 k}\left(\right.$ from $R_{2}$ and $R_{3}$ ), and discards the one with the lower

\section{value of $I_{d_{k}}$.}

The identifier is required since $G_{\text {Icurr }}$ changes with time: whenever robot $R_{i}$ receives $G_{\text {Icurr }}$, it stores the goal (after having computed its position with respect to $F_{i}$ ) together with its identifier $\operatorname{Id}_{1 \mathrm{k}}$. Next, when communicating it to another robot $R_{j}$, it marks $G_{\text {Icurr }}$ with $\operatorname{Id}_{1 \mathrm{k}}-1 . R_{j}$ is thus aware of the fact that it has not received $G_{\text {Icurr }}$ directly from $R_{l}$, but from another robot that directly communicates with $R_{l}$. Notice that the identifier received by $R_{j}$ still identifies unequivocally $R_{I}$ 's $\mathrm{k}^{\text {th }}$ goal (since $\mathrm{k}=1+\left(\mathrm{Id}_{1 \mathrm{k}}-1\right) / \mathrm{M}$ ) but, on the other side, it also provides an estimate of the quality of the information, since it measures the number of robots by which the information has been manipulated. This is important because the computation of the transformation matrix is affected by noise in reality, and it is thus possible that $R_{j}$ checks the position of $G_{\text {Icurr }}$ in the wrong place. To reduce the effects of noise, each robot will prefer to receive $G_{\text {Icurr }}$ directly from $R_{l}$ whenever possible. The number of coordinates transformations that have been performed on $G_{\text {Icurr }}$ before the information reached $R_{j}$ can be computed as $\mathrm{Nr}_{\mathrm{ik}}=\left(\mathrm{Id}_{1 \mathrm{k}}-(\mathrm{k}-1) * \mathrm{M}\right)$, where $\mathrm{Nr}_{\mathrm{ik}}=\mathrm{M}$ only if the goal has been directly communicated by $R_{l}$, and $\mathrm{Nr}_{\mathrm{ik}}=1$ if the information has been manipulated by all the robots before reaching $R_{i}$. If $R_{i}$ receives multiple reports about $G_{\text {Icurr }}$, it accepts the new goal as a valid one only if its identifier is greater than or equal to the one that had been previously stored for that robot's goal (Figure 5a). That 
is, if the goal is more recent (updated information) or if it comes from a robot which is closer to $R_{l}$ (better quality information).

\subsection{Rules 3 and 4: check goals'visibility and reflect visibility.}

Each robot $R_{i}$ that knows $G_{\text {Icurr }}$ checks its visibility $\left(G_{\text {lcurr }} \in V\left(R_{i}\right)\right)$; furthermore, it communicates the result to each $\operatorname{robot} R_{j} \in V\left(R_{i}\right)$. Specifically, when $R_{i}$ sees $G_{\text {Icurr }}$ it transmits the following information:

1. $\operatorname{Id}_{1 \mathrm{k}}=$ the identifier of the goal that it has seen (which can differ from $R_{l}$ 's current goal because of delays in inter-robot communication).

2. $\mathrm{D}_{1 \mathrm{i}}$, the Euclidean distance between $R_{i}$ and $G_{\text {Icurr }}$.

We have stated that, if $G_{\text {lcurr }} \in V\left(R_{i}(t)\right)$ and $R_{i}(t) \in V\left(R_{j}(t)\right)$, we let $R_{j}$ infer that $G_{\text {lcurr }} \in V_{R}\left(R_{j}(t)\right)$. However, when $R_{j}$ wants to communicate to another robot that it can see $R_{l}$ 's goal, it computes its distance to $G_{\text {Icurr }}$ as $\mathrm{D}_{1 \mathrm{j}}=$ dist $\left(R_{i}, R_{j}\right)+\mathrm{D}_{1 \mathrm{i}}$ which is clearly larger that the distance computed by $R_{i}$, the robot that can directly perceive the goal.

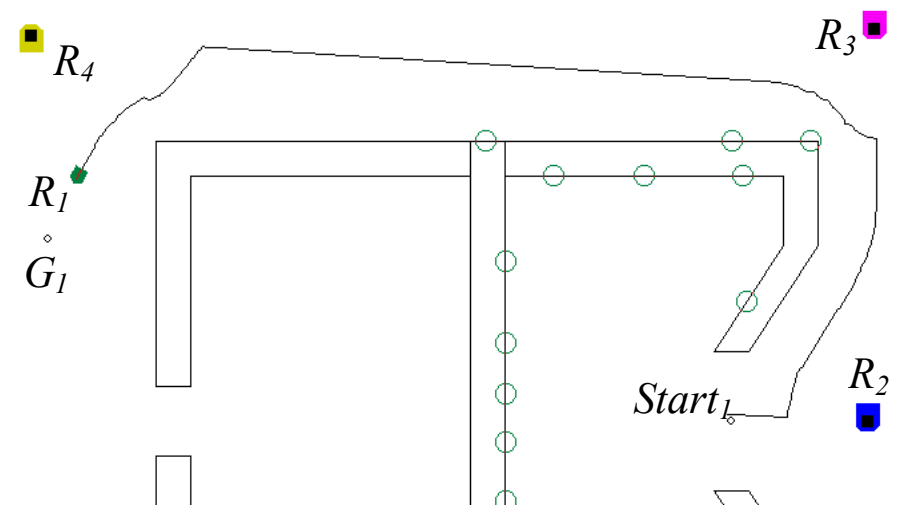

Figure 6. $R_{1}$ 's trajectory is shown (in simulation) when it receives information about its goal from $\boldsymbol{R}_{2}$, $R_{3}$, and $R_{4}$

More generally when $R_{j}$ receives information from different robots, each declaring to see $G_{\text {Icurr }}$, it listens only to the robot that guarantees the shortest path to the goal, thus avoiding loops in communication (see Figure 5b). Next, when re-transmitting the information, it computes its distance to the goal according to the previous rule. Finally, $R_{I}$ 
accepts only the information that is associated with the shortest distance and discards the others, therefore heading towards the robot that provides the shortest path to its goal (guaranteed if the environment is static).

As a consequence, if suddenly $R_{l}$ perceives its own goal directly, it starts heading toward it since no other information can have a lower value as it is guaranteed to be the shortest distance. Figure 6 shows the behavior of the system for a very simple simulated case.

\section{MOVING ROBOTS AND 'GHOST ROBOTS'.}

Up to now we suppose a team of $\mathrm{M}$ robots of which only $R_{l}$ was given a navigation problem and therefore requested to move in the environment, while the other robots $\left(R_{2}\right.$ to $\left.R_{M}\right)$ were stationary. One could try to compute, for a given environment, the minimum number of fixed support robots that is required to guarantee, for each pair of

points in the environment (say $R_{l}$ and $G_{\text {Icurr }}$ ), that the following expression is true: $G_{\text {lcurr }} \in V_{R}\left(R_{1}(t)\right.$ ). The problem seems very similar to coverage problems that have been deeply studied in the literature: art gallery problems [14] or the pursuit-evasion problem [15]. However, neither of these approaches fits very well into the motivation of our research. First of all, we aim at designing cooperation strategies for robots that have a very limited knowledge (or no knowledge at all) of the environment and therefore rely on local navigation algorithms to find their way to the target. Since the environment is unknown, it is not possible to compute the minimum number of fixed support robot that are required to fully cover the environment, nor to determine a motion strategy that allows in different times to displace M-1 support robots such as to make $R_{l}$ 's current goal visible from $R_{l}$ 's current position. Furthermore, even if computable, this number could be very high, and it appears to waste resources by using those robots only to help one robot to safely carry out its tasks.

Thus, we choose a different approach, and let all robots move in the environment while carrying out their own activities; that is, instead of determining a motion strategy or a spatial distribution that allow robots to help each other, we focus our attention on finding a strategy that allows them to do their best in helping each other whenever they have a chance. However, if we let the robots move without coordinating them, it is intuitive that the probability for the expression $G_{1 \text { curr }} \in V_{R}\left(R_{1}(t)\right)$ to be true becomes very low (and depends on the complexity of the environment, the number of robots involved, and the task of each robot). Thus, we choose to introduce a new visibility function, which we call delayed visibility $\mathrm{V}_{\mathrm{D}}(R)$. We wish to anticipate that, because of the following 
extension, we lose one of the properties of the previous approach, which was purely reactive in the sense that it did not require memory except for storing the two values $\mathrm{Id}_{\mathrm{ik}}$ and $\mathrm{D}_{\mathrm{i}}$ (the identifier of the goal and the length of the path), both required only for arbitrating between different solutions and choosing the optimum one. While previously we could imagine that at time t, $R_{I}$ perceived its own goal reflected by $R_{2}$, in the following this will no longer be true: $R_{I}$ still behaves as if it can see its own goal reflected in another robot which has seen its goal, but it is no longer guaranteed, even in a static environment, to reach its goal while following a finite set of straight trajectories. The algorithm can be described as follows (Rules 1 to 5 are the same as the previous case; we just need substituting $\mathrm{V}_{\mathrm{R}}(R)$ with the new function $\left.\mathrm{V}_{\mathrm{D}}(R)\right)$ :

\subsection{Rules 1 and 2: share goals with other robots.}

Once again, when a generic robot $R_{l}$ shares its own $\mathrm{k}^{\text {th }}$ goal, it marks it with a unique identifier $\mathrm{Id}_{1 \mathrm{k}}$, whose value is computed as in the previous case. Each robot $R_{j}$ accepts $G_{l k}$ as a valid goal only if its identifier $\mathrm{Id}_{1 \mathrm{k}}$ is greater than or equal to the one that it had been previously stored for $R_{l}$. Notice that now each robot needs to remember many different goals (a maximum of M-1 goals) while it is moving in the environment towards its own goal, while previously this was not needed since each robot received continuously updated information from all the robots that it could see. However this appears not to be a major problem, at least from the computational complexity and the memory requirements (both being linear with respect to the number of robots).

\subsection{Rules 3 and 4: check goals' visibility and reflect visibility.}

When $R_{j}$ communicates to $R_{i}$ that $G_{1 \text { curr }} \in V_{D}\left(R_{j}\right)$, it transmits the identifier of the goal $\operatorname{Id}_{1 \mathrm{k}}$ together with $\mathrm{D}_{1 \mathrm{j}}$ (however, because of the introduction of $\mathrm{V}_{\mathrm{D}}(R)$, the estimate of the distance to $G_{\text {Icurr }}$ is now different; it will be described in the following paragraph). Thus $R_{i}$ estimates its distance to the goal as $\mathrm{D}_{1 \mathrm{i}}=\operatorname{dist}\left(R_{i}, R_{j}\right)+\mathrm{D}_{1 \mathrm{j}}$ and stores this value in its memory. If at a given time a third robot $R_{k}$ comes in sight and communicates that $G_{\text {lcurr }} \in V_{D}\left(R_{k}\right), R_{i}$ compares the stored distance $\mathrm{D}_{1 \mathrm{i}}$ with $\operatorname{dist}\left(R_{i}, R_{k}\right)+\mathrm{D}_{1 \mathrm{k}}$ and attends to the new information only if it guarantees a shorter path to the goal. Finally, $R_{l}$ accepts only the information that is associated to the shortest distance and discards the rest, heading towards the robot that provides the shortest path to its goal. 
However, the same rule is now true even when the information $G_{\text {lcurr }} \in V_{D}\left(R_{i}\right)$ is communicated by the same robot $R_{i}$, but at two different times (which happens frequently since robots are navigating in the environment towards their own goals). Suppose that at time $\mathrm{t}_{1}$ robot $R_{2}$ provides a path with distance $\mathrm{D}_{12}\left(\mathrm{t}_{1}\right)$ (Figure 7 ). $R_{I}$ stores in its memory $\mathrm{D}_{11}\left(\mathrm{t}_{1}\right)=\operatorname{dist}\left(R_{l}\left(\mathrm{t}_{1}\right), R_{2}\left(\mathrm{t}_{1}\right)\right)+\mathrm{D}_{12}\left(\mathrm{t}_{1}\right)$. If at time $\mathrm{t}_{2}\left(\mathrm{t}_{2}>\mathrm{t}_{1}\right) R_{2}$ is farther both from the goal and from $R_{l}$, it provides a path which is given a score $\mathrm{D}_{12}\left(\mathrm{t}_{2}\right)$ such that $\operatorname{dist}\left(R_{l}\left(\mathrm{t}_{2}\right), R_{2}\left(\mathrm{t}_{2}\right)\right)+\mathrm{D}_{12}\left(\mathrm{t}_{2}\right)>\mathrm{D}_{11}\left(\mathrm{t}_{1}\right)$. As a consequence, $R_{l}$ continues to head toward the location in which $R_{2}$ was at time $\mathrm{t}_{1}$, even if $R_{2}$ is no more there. We can imagine that it continues to head toward a 'ghost robot' that provides the shortest path to the goal (as an aside: the ancient Greeks used the same word for 'ghost' and 'mirror', eidolon). Notice that this reduces the reactivity of the system, since $R_{I}$ now needs to remember the position $R_{2}\left(\mathrm{t}_{1}\right)$ in which $R_{2}$ was located when it promised the best path, instead of being purely controlled by its own sensory inputs. This seems to be necessary whenever we allow robots to move, since otherwise $R_{1}$ would have the tendency to follow $R_{2}$ even if it is heading in the opposite direction with respect to $G_{\text {Icurr }}$. Moreover, if $R_{l}$ is able to distinguish some interesting features in the environment and to use such cues for navigating towards $R_{2}\left(\mathrm{t}_{1}\right)$, the consideration regarding keeping the focus of attention are still valid.

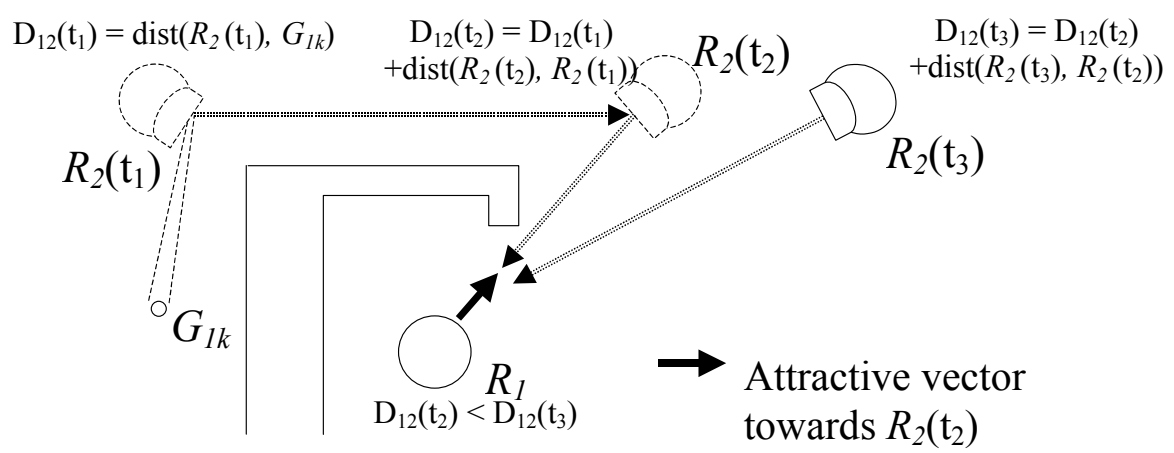

Figure 7. $R_{2}\left(\mathrm{t}_{2}\right)$ provides a shorter path than $\boldsymbol{R}_{2}\left(\mathrm{t}_{3}\right)$. As a consequence, $\boldsymbol{R}_{1}$ continues to head toward the location in which $R_{2}$ was at time $\mathrm{t}_{2}$, even if $R_{2}$ is no longer there.

Finally notice that, since $R_{l}$ heads towards $R_{2}$ even if $R_{2}$ is not currently seeing $G_{\text {Icurr }}, R_{l}$ is no longer guaranteed to perceive the goal (or finding further help for reaching the goal) when it has reached $R_{2}$ 's position. To the contrary, in most cases, it will have to start again to explore the environment by relying on its own navigation algorithm $A$, 
possibly from a 'better position'. We will attempt to demonstrate experimentally that this position is really a better one.

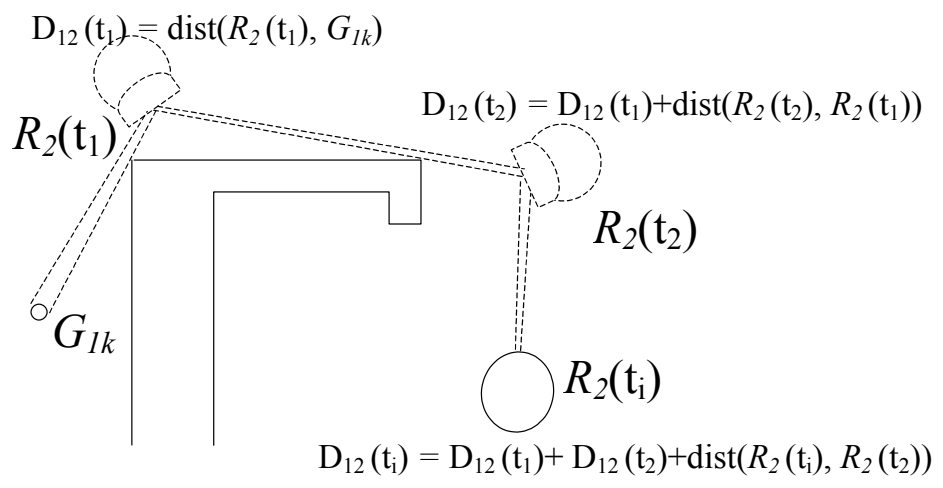

Figure 8. Computation of the distance to the goal when $R_{2}$ is moving.

\subsection{Computation of the distance from the goal}

Since $R_{i}$ is moving, and not constantly in sight of $G_{\text {lcurr }}$, we need to explain how it computes the distance $\mathrm{D}_{1 \mathrm{i}}$ when it communicates $G_{\text {lcurr }} \in V_{D}\left(R_{i}\right)$. When $R_{i}$ directly perceives $G_{\text {Icurr }}$ (i.e., $\left.G_{\text {lcurr }} \in V\left(R_{i}\right)\right), \mathrm{D}_{1 \mathrm{i}}$ is computed at each time step as $\operatorname{dist}\left(R_{i}, G_{\text {Icurr }}\right)$ as usual; however, when $G_{\text {Icurr }}$ is no longer visible $R_{i}$ recalls the last position $R_{i}\left(\mathrm{t}_{1}\right)$ from which $G_{\text {Icurr }}$ was visible and computes $\mathrm{D}_{1 \mathrm{i}}\left(\mathrm{t}_{1}\right)=\operatorname{dist}\left(R_{i}\left(\mathrm{t}_{1}\right), G_{\text {Icurr }}\right)$ and stores the result in memory (Figure 8). In the following, $R_{i}$ checks if $R_{i}\left(t_{1}\right) \in V\left(R_{i}\right)$ and computes $\mathrm{D}_{1 \mathrm{i}}$ as $\mathrm{D}_{1 \mathrm{i}}\left(\mathrm{t}_{1}\right)+\operatorname{dist}\left(R_{i}, R_{i}\left(\mathrm{t}_{1}\right)\right)$. We can again imagine a 'ghost robot' $R_{i}\left(\mathrm{t}_{1}\right)$ which sees the goal and transmits the location to $R_{i}$, which can then estimate its own distance to the goal (Figure 8). If, at time $\mathrm{t}_{2}\left(\mathrm{t}_{2}>\mathrm{t}_{1}\right)$ a $R_{i}\left(\mathrm{t}_{1}\right)$ is also no longer visible, $R_{i}\left(\mathrm{t}_{1}\right)$ and $\mathrm{D}_{1 \mathrm{i}}\left(\mathrm{t}_{1}\right)$ are substituted in memory with $R_{i}\left(\mathrm{t}_{2}\right)$ and $\mathrm{D}_{1 \mathrm{i}}\left(\mathrm{t}_{2}\right)$, and $R_{i}$ computes $\mathrm{D}_{1 \mathrm{i}}$ as $\mathrm{D}_{1 \mathrm{i}}\left(\mathrm{t}_{2}\right)+\operatorname{dist}\left(R_{i}, R_{i}\left(\mathrm{t}_{2}\right)\right)$.

\subsection{Complexity of the algorithm}

If we consider a team composed of $\mathrm{M}$ robots, each robot $R_{j}$ needs to remember a maximum of $\mathrm{M}$ goals or 'ghost robots' together with the associated $\mathrm{Id}_{\mathrm{ik}}$ and $\mathrm{D}_{\mathrm{ij}}$ (corresponding to $R_{j}$ 's goal plus the goals of all the robots that it has seen) and to periodically check their visibility. This guarantees linear complexity and memory occupancy for the algorithm. 
Whenever two robots meet, a communication mechanism with a bandwidth of order $\mathrm{O}(\mathrm{M})$ is needed since each of the two robots communicates a maximum of $M$ terms $\left(G_{i k}, \alpha, I_{i k}\right)$ and a maximum of $M$ pairs $\left(\operatorname{Id}_{i k}, D_{i k}\right)$. If $M$ robots are in sight and communicate with each other at the same time, the bandwidth required is $\mathrm{O}\left(\mathrm{M}^{2}\right)$.

\section{AGENTS COMMUNICATING THEIR INTERNAL STATE.}

In [8] a formal analysis is presented regarding the benefits provided by different types of communication in improving agents' behavior in different multi-agent scenarios. More specifically, agents explicitly sharing their goals and internal state are compared with agents making use only of implicit communication in three basic tasks (foraging, consuming, and grazing) that can be taken as the basis for building more general and complex tasks. One motivation is to provide a guide for the design of multi-agent systems, and to verify if the use of a more complex and therefore expensive language (required for sharing goals) is really more effective than communicating only one bit of information to encode the internal state, (analogous to what biologists call display behavior) or by avoiding any explicit communication altogether. Following these design guidelines, we provide robots with another mechanism for helping each other to achieve their own goal, which relies on the communication of their internal states encoded through a single bit of information. We want to verify if it is possible to use this different mechanism (the communication of the internal state) to help robots succeed in the cases where the previous mechanism fails and, more generally, to improve the overall performance of the system.

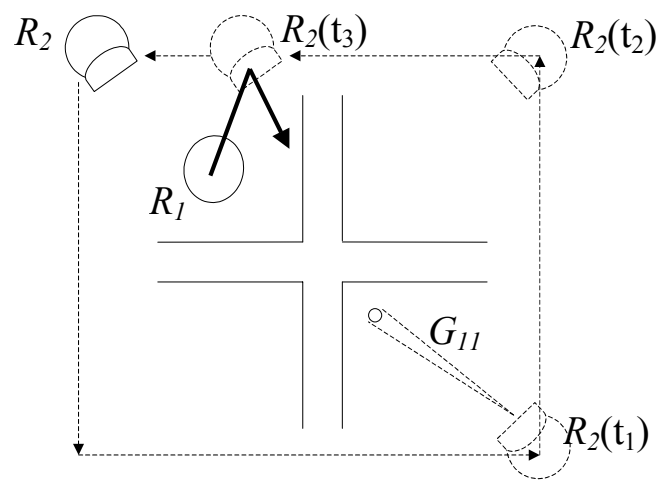

Figure 9. Once $R_{1}$ has reached $R_{2}\left(\mathrm{t}_{3}\right)$, it is not in a better position for finding a path to its goal, and gets trapped again in the local minimum. 
A typical case where the goal-sharing mechanism, presented earlier in this paper, fails appears in Figure 9. $R_{I}$ 's goal is to reach $G_{I I}$, while $R_{2}$ is simply patrolling the area. Since $R_{2}$ has seen $G_{I I}$ when in $R_{2}\left(\mathrm{t}_{1}\right), R_{l}$ heads towards $R_{2}\left(\mathrm{t}_{3}\right)$. However, once $R_{l}$ has reached $R_{2}\left(\mathrm{t}_{3}\right)$, it is no longer in a better position for finding a path to its goal, and gets trapped into the local minimum.

The new mechanism proposed is not dependent on the local algorithm $A$ that each robot uses for finding a path to the goal. Instead, the following strategy works with all generic algorithms $A$ for which it is possible to define an estimate of its performance in conducting the robot to the goal. In a simple potential field approach, for example, it is easy for the robot to evaluate its progress to the goal and to realize when it is stuck in a local minimum (although it is not that simple to provide the robot with a strategy for escaping the local minimum!). Obviously, depending on the particular algorithm adopted, different metrics can be chosen for synthesizing the concept of 'being in trouble'. However, a very simple one can be chosen independently from the navigational algorithm adopted: if the robot is taking too much time in reaching its goal, it is very likely that the robot is having 'serious trouble' in achieving its mission.

With respect to the previous case, the rules required for robot cooperation are even simpler:

1. Evaluate my progress: Each robot stores a Boolean variable $\operatorname{Tr}_{\mathrm{i}}$ : $\operatorname{Tr}_{\mathrm{i}}$ is false if the robot thinks that it is progressing well towards the goal (the robot is 'not in trouble'), true if it realizes that it is not making progress (the robot is 'in trouble').

2. Share state: Each robot $R_{i}$ communicates to each robot $R_{j} \in V\left(R_{i}\right)$ its current state $\operatorname{Tr}_{\mathrm{i}}$.

3. Head to helper: If robot $R_{i}$ is 'in trouble' and $R_{j} \in V\left(R_{i}\right)$ is 'not in trouble', $R_{j}$ temporarily becomes $R_{i}$ goal; that is, $R_{i}$ starts heading towards $R_{j}$ until it realizes that it is no longer 'in trouble' or it is eventually able to perceive its own goal.

Once again, $R_{i}$ believes temporarily that $R_{j}$ is in a better area of the environment for reaching its own goal, and therefore heads towards $R_{j}$ to increase its own performance. Obviously, the fact $R_{j}$ is making progress towards its own goal does not imply following $R_{j}$ will help $R_{i}$ to reach a better position. However, if $R_{j}$ is a support robot that patrols the environment, experiments confirm that the previous assertion is true in many cases (an example is shown in Figure 10: it will be described in details in the following). 
Particular attention should be paid to the updating rule of the variable $\operatorname{Tr}_{\mathrm{i}}$, which defines the state of the $\mathrm{i}^{\text {th }}$ robot. In order to update $\mathrm{Tr}_{\mathrm{i}}$, each robot stores in memory a pair of variables

1. $\operatorname{Pr}_{\mathrm{i}}$, which increases at each computation step of the algorithm if the robot is not making progress towards the goal; it decreases otherwise.

2. $\mathrm{Cr}_{\mathrm{i}}$, which establishes an upper limit on the value of $\operatorname{Pr}_{\mathrm{i}}$.

$\operatorname{Tr}_{\mathrm{i}}$ changes to true when $\operatorname{Pr}_{\mathrm{i}}=\mathrm{Cr}_{\mathrm{i}}$ and changes back to false when $\operatorname{Pr}_{\mathrm{i}}=0$. Moreover, each time that $\operatorname{Tr}_{\mathrm{i}}$ changes to false, we increment a third value $\mathrm{Nt}_{\mathrm{i}}$ (whose initial value is 1 ), which counts the number of transitions between the state 'in trouble' and the state 'not in trouble' that have taken place in the robot. When the robot is not making progress towards the goal, $\mathrm{Pr}_{\mathrm{i}}$ is increased by $\mathrm{Nt}_{\mathrm{i}}$; when the robot is progressing towards the goal, $\mathrm{Pr}_{\mathrm{i}}$ is decreased by 1. Finally the upper bound to the value of $\mathrm{Pr}_{\mathrm{i}}$ is set to $\mathrm{Nt}_{\mathrm{i}} \mathrm{Cr}_{\mathrm{i}}$.

This allows the behavior depicted in Figure 10 (in a simulated environment). Since it has no direct means (in this case) of coming out of the local minimum, $R_{l}$ remains stuck in its start position Start $_{1}$ (we have seen that, even if it communicates its goal $G_{11}$ to $R_{2}$, this does not provide any help). While $R_{1}$ is stuck, the value of $\operatorname{Pr}_{\mathrm{i}}$ increases by $\mathrm{Nt}_{\mathrm{i}}=1$ at each computation step until it reaches the maximum value $\mathrm{Cr}_{\mathrm{i}}$; when $R_{2}$ (which is progressing towards its goal $G_{22}$ ) comes in sight, $R_{1}$ starts heading towards it since $\operatorname{Tr}_{1}=$ true and $\operatorname{Tr}_{2}=$ false. However, since $R_{1}$ is now progressing towards its goal (by setting $G_{i c u r r}=R_{2}$ ), $\operatorname{Pr}_{1}$ decreases to zero, and $\operatorname{Tr}_{1}$ changes to false; $R_{I}$ stops following $R_{2}$ and gets again trapped in the local minimum while heading towards $G_{11}$.

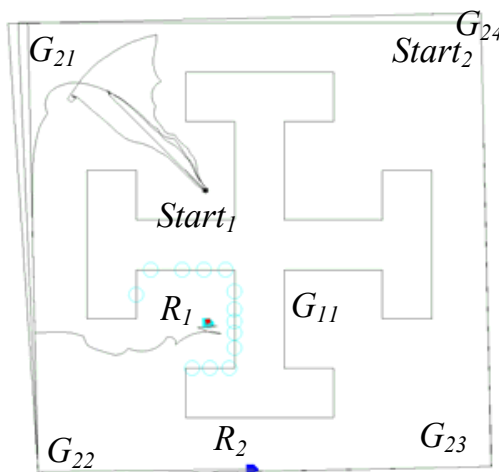

a)

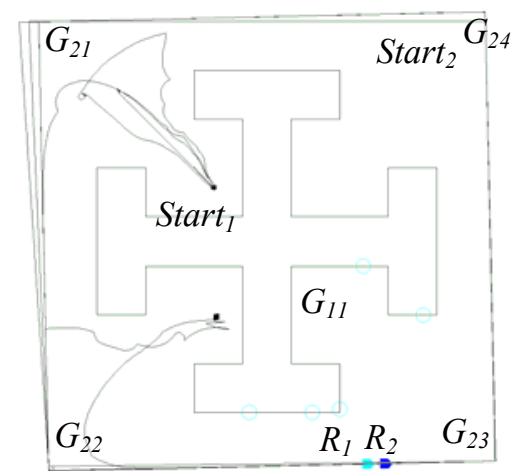

b)

Figure 10. Thanks to the help of $R_{2}, R_{1}$ finally manages to find a path to the goal. 
When $R_{2}$ comes in sight again, $\operatorname{Pr}_{\mathrm{i}}$ probably has a higher value, since it has been incremented with a higher delta $\left(\mathrm{Nt}_{\mathrm{i}}=2\right)$ and the upper bound is set to a higher value $\left(2 \mathrm{Cr}_{\mathrm{i}}\right)$. This time $R_{l}$ follows $R_{2}$ for a longer time before heading again towards its own goal. After several attempts, it can be seen that $R_{l}$ finally manages to make its path to the goal (Figure 10).

\section{EXPERIMENTAL RESULTS}

The system has been extensively tested in the simulated MissionLab [16] environment. Experiments have been performed in two different scenarios (Figures 11 and 13). Both scenarios represent typical office-like indoor environment of different complexity: $R_{l}$ is given an exploration task (it has to reach target positions which are located in the middle of the rooms) whilst other robots are deployed in the environment with the purpose of patrolling the corridors and supporting $R_{l}$ in its task. In particular:

- $R_{l}$ is assigned exploration tasks, each corresponding to a set of 200 randomly chosen targets to be reached in sequence. The same task is executed by providing $R_{l}$ with different navigation algorithms $A$ : 1) a standard potential field algorithm, 2) a potential field algorithm to which the Avoid Past schema [17] has been added, and 3) the VisBug21 algorithm [7]. Moreover, each navigation algorithm is used to execute 5 exploration tasks, by randomly choosing different sets of targets to be reached. This allows us to compute the average number of motion steps required by $R_{l}$ to explore the environment with a given navigation algorithm together with its standard deviation.

- In order to test the coordination mechanism, the average number of motion steps required is first computed when no external help is available; next, the same exploration task is executed by adding an increasing number of supporting robots $\left(R_{2}, R_{3}\right.$, and $\left.R_{4}\right)$ into the environment with the purpose of helping $R_{1}$ to accomplish its mission

Notice that all the navigation algorithms considered are local algorithms, i.e. they do not rely on global a-priori information about the environment. In particular: 1) does not guarantee the robot to reach its target without the help of other robots because of the presence of local minima in the potential fields, 2) proves to be effective to help the robot to explore the environment and 3) is guaranteed to find a path to the goal (whenever it exists) even when no helping robots are present. Thus, in the case of 1), the graphs in Figures 12 and 14, do not show any result when only $R_{l}$ is present. For analogous reasons, in the case of 1) we implement both the state-sharing strategy described in the 
previous section and the goal-sharing strategy described in sections II to IV. On the opposite, in the case of 2) and 3) only the goal-sharing mechanism is required. Finally notice that, at each motion step, the distance traveled by the simulated robots is fixed, since only the direction of motion changes: as a consequence, the number of motion steps required to execute an exploration task is directly proportional to the length of the path followed during the task.

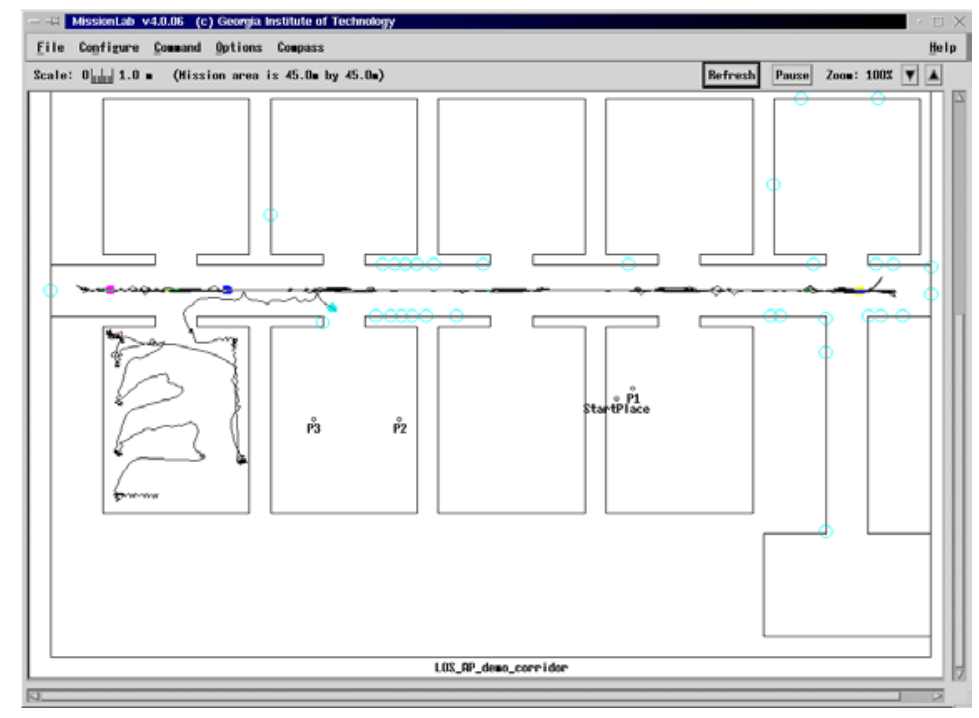

Figure 11. First simulated experimental scenario.

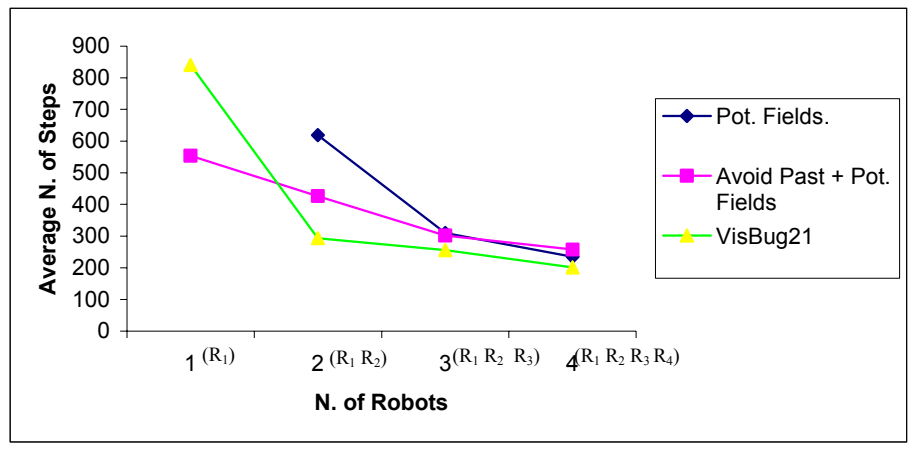

Figure 12. The results of the experiments performed in the first scenario.

In the two graphs in Figures 12 and 14 the average number of motion steps required by $R_{I}$ to complete exploration tasks is plotted versus the number of robot present in the environment: each curve corresponds to the different navigation algorithm. Notice that, in all cases, the performance significantly increases as we add more helping robots. In particular, in Figure 12 it can be seen that the number of motion steps required by the VisBug algorithm 
reduces from more than 800 to about 300 when adding just one support robot. Next, it decreases to about 250 when all three helping robots have been added. As regard to the other algorithms (potential field and Avoid Past), the improvement in performance is less evident in this very simple scenario: the number of computation steps decreases from about 550 to about 250 for Avoid Past and from about 630 to about 230 for the potential field algorithm. Table 1 shows in details the same results in fig. 12, by reporting the standard deviation corresponding to each value shown in the graph (standard deviation is expressed as a percentage of the average number of motion steps and provides a measure of the significance of the data).

\begin{tabular}{l|c|c|c|c|} 
Av.Past. & $\mathrm{R} 1$ & $\mathrm{R} 1,2$ & $\mathrm{R} 1,2,3$ & $\mathrm{R} 1,2,3,4$ \\
\hline Av. Steps & 554,2 & 426,2 & 302,0 & 257,2 \\
St.Dev. & $11,7 \%$ & $8,1 \%$ & $14,2 \%$ & $5,6 \%$ \\
\hline VisBug & & & & \\
\hline Av. Steps & 840,6 & 294,0 & 255,4 & 201,2 \\
St.Dev. & $7,6 \%$ & $6,6 \%$ & $6,1 \%$ & $3,7 \%$ \\
\hline Pot. Fields & & & & \\
\hline Av. Steps & & 618,6 & 310,0 & 235,2 \\
St.Dev. & & $3,6 \%$ & $10,0 \%$ & $5,4 \%$
\end{tabular}

\section{Table 1. Results of the experiments performed in the first scenario.}

Finally notice that, in this scenario, the performance of the system seems not to depend on the particular algorithm adopted when we add more than two robots (the curves in fig. 12 becomes very similar when N. of Robots $>=3$ ); since the environment is very simple, the trajectory followed by $R_{l}$ depends more on the paths suggested by $R_{2} R_{3}$, and $R_{4}$ than on the algorithm $A$. 


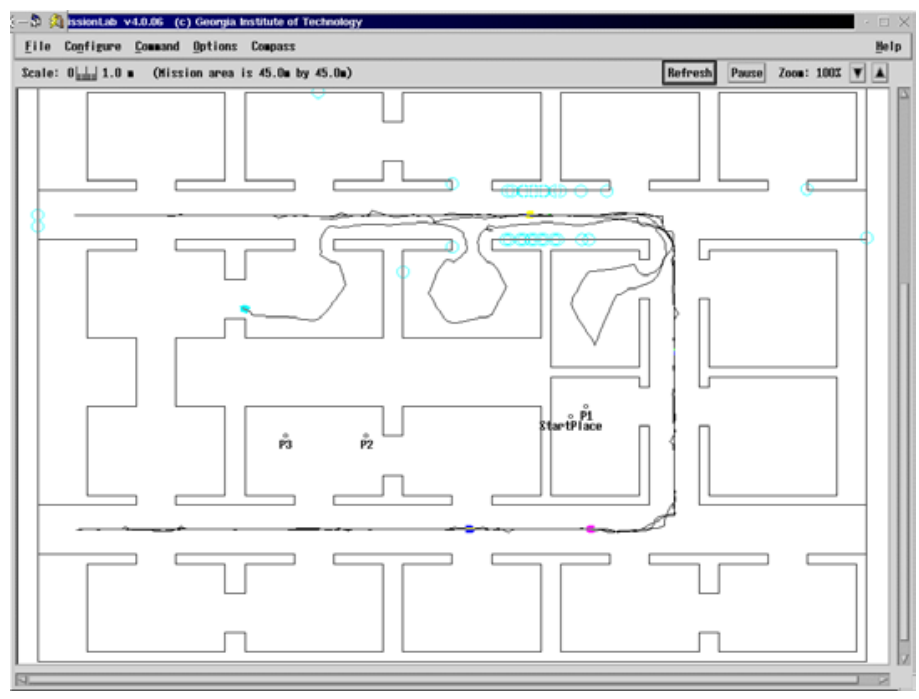

Figure 13. Second simulated experimental scenarios.

The situation is different in the second, more complex scenario, as it can be seen in Figure 14. Even when three helping robots are present, the influence of the navigation algorithm $A$ is still important in determining the path followed by $R_{l}$, and consequently the number of computation steps required is different in the three cases. In particular, it can be noticed that VisBug is more efficient to find a path to the goal: the corresponding curve stays always below the two other curves, independently from the number of support robots which have been deployed in the environment. However, we are not interested in comparing the three algorithms, since their performance depends on the environment chosen and the tuning of some parameters. Instead, we want to show that the performance of a given algorithm improve when adding support robots: in this scenario, the number of steps required by VisBug decrease from about 500 to about 370, the steps required by Avoid Past decrease from about 1130 to about 680, and the steps required by the potential fields algorithm decrease from about 1250 to about 900 . Table 2 shows in details the same results in fig. 14, by reporting the standard deviation corresponding to each value shown in the graph 


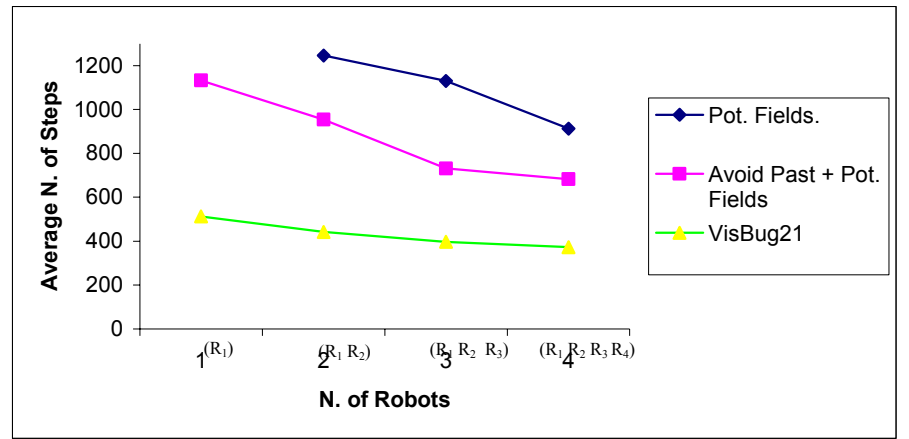

Figure 14. The results of the experiments performed in the second scenario.

\begin{tabular}{l|c|c|c|c} 
Av.Past. & $\mathrm{R} 1$ & $\mathrm{R} 1,2$ & $\mathrm{R} 1,2,3$ & $\mathrm{R} 1,2,3,4$ \\
\hline Av. Steps & 1132,2 & 953,6 & 731,8 & 682,6 \\
St.Dev. & $4,5 \%$ & $6,4 \%$ & $7,9 \%$ & $6,9 \%$ \\
\hline VisBug & & & & \\
\hline Av. Steps & 513,6 & 441,8 & 397,4 & 373,4 \\
St.Dev. & $10,5 \%$ & $12,0 \%$ & $9,0 \%$ & $10,3 \%$ \\
\hline Pot. Fields & & & & \\
\hline Av. Steps & & 1246,4 & 1130,4 & 913,6 \\
St.Dev. & & $5,9 \%$ & $6,7 \%$ & $4,7 \%$
\end{tabular}

Table 2 Results of the experiments performed in the second scenario.

In general, experiments show that the efficiency of all the algorithms is significantly improved when adding the simple coordination mechanism that has been presented in the previous Sections.

\subsection{Adding noise to simulations}

Up to now, we showed the performance of a system with perfect sensing and navigation capabilities: however, in a realistic implementation, it is required to deal with the problem of uncertain information. In general, we devise two main possible sources of uncertainty:

1. uncertainty in localization, i.e. each robot has only an approximate estimate of its position in the world

2. uncertainty in sensing, i.e. each robot has only an approximate estimate of the position of other robots which are in line-of-sight.

As regard to point 1, it should be noticed that uncertainty in localization is a general problem for navigation, and not a specific problem of the presented approach. Obviously, if robot $R_{l}$ wants to reach a target location in the environment, it has to know with a certain degree of accuracy its own position in the world with respect to a absolute 
reference frame; otherwise, it will reach a wrong location or, even, it will not be able to reach its target at all. The presented approach is not an exception. However, as it has been already explained in section III.A, only $R_{l}$ is responsible for knowing with accuracy the place where it needs to go; other robots, even if they are completely lost in the environment, are still fully functional in helping $R_{l}$, since they do never rely on the absolute reference frame to retrieve information about $R_{I}$ 's goal visibility.

Thus, localization plays a role in the system only when robot $R_{l}$ is heading towards a 'ghost robot' (see Figure 7): if at time $\mathrm{t}>\mathrm{t}_{2} R_{l}$ is heading towards the position which was occupied by $R_{2}$ at time $\mathrm{t}_{2}$ (and, in the meanwhile, $R_{2}$ has moved), $R_{l}$ must rely on its own positioning system to reach $R_{2}\left(\mathrm{t}_{2}\right)$. However, $R_{I}$ path is by definition a straight one, whose length is upper bounded by the radius of $R_{l}$ 's visibility area. Since the visibility radius is small in most indoor applications and the path is a straight one, we assume odometry to be adequate to guarantee $R_{2}\left(\mathrm{t}_{2}\right)$ reachability. As a consequence of all this considerations, we choose to ignore localization uncertainty in our experiments.

As regard to point 2, we assume that each robot is equipped with a vision system, and it is therefore able to detect teammate robots in the environment (to make this task easier, one could put a well identifiable marker on each robot) and to retrieve teammates' positions by means, for example, of a inverse perspective mapping algorithm. Given this assumptions, uncertainty in sensing could be the consequence of two major causes: bad lighting conditions or partial occlusions. Since the effects of lighting conditions on a vision system are very complex to be modeled, we focus on the occlusion problem. In particular, when robot $R_{1}$ sees $R_{2}$, we assume that $R_{2}$ 's position with respect to $F_{1}$ (see Section III.A) is first computed in polar coordinates. Given $R_{2}$ 's real distance $d_{r}$ and angle $\alpha_{r}$, we define the perceived distance $d_{p}$ and angle $\alpha_{p}$

$$
\begin{aligned}
& d_{p}=d_{r}+d_{r} d_{\text {noise }} \\
& \alpha_{p}=\alpha_{r}+\tan ^{-1}\left(\frac{\text { robotsize }}{2 d_{r}}\right) \alpha_{\text {noise }}
\end{aligned}
$$

$d_{\text {noise }}$ is stochastic variable with a uniform distribution in the interval [- $-\mathrm{err}_{\max } ;+\operatorname{err}_{\max }$, which introduces an error in the perceived distance: we assume that the maximum error in $d_{p}$ depends on the real distance $d_{r}$, i.e. the farther is the robot, the bigger is the error. $\alpha_{p}$ is a stochastic variable with a uniform distribution in the interval $[-1 ;+1]$ : notice 
that, since we assume that all errors are due to partial occlusions, we are allowed to put an upper bound on the maximum error on $\alpha_{p}$ (as defined in equation 2 and shown in Figure 15).

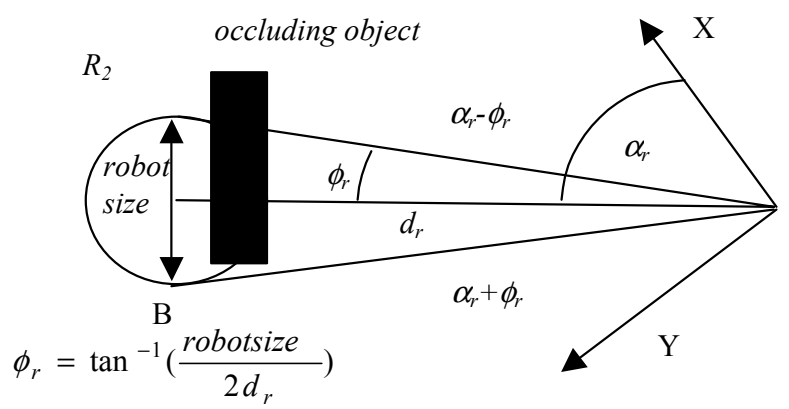

Figure 15. Because of a partial occlusion, $R_{2}$ 's angle $\alpha_{p}$ differs from the real angle $\alpha_{r}$.

The experiments described in Figures 11 to 14 have been performed again after adding noise to the simulated vision sensor: Figures 16 and 17 correspond, respectively, to the environments in Figures. 11 and 13. The graphs show the average number of motion steps required by $R_{l}$ to reach its goals plotted versus err $_{\max }$ (when all the supporting robots $R_{2}, R_{3}$, and $R_{4}$ are present). Finally, the rightmost value of each plot corresponds to the case in which no supporting robots are present.

Notice that, when $\operatorname{err}_{\max }$ increases, the performance of the system decreases for every navigation algorithm. However, in Figure 16, even when $\operatorname{err}_{\max }=0.4$ (corresponding to a maximum error which is about half the real distance $d_{r}$ ) the average number of steps required by $R_{l}$ to reach its goal is still lower than the case in which no supporting robots are present. On the opposite, in Figure 17 (corresponding to a more complex environment) it can be noticed that, when err $_{\max }$ increases, support robots become very soon almost useless. In particular, the performance of VisBug when $\operatorname{err}_{\max }$ increases are even worse than the " $R_{l}$ alone" case. 


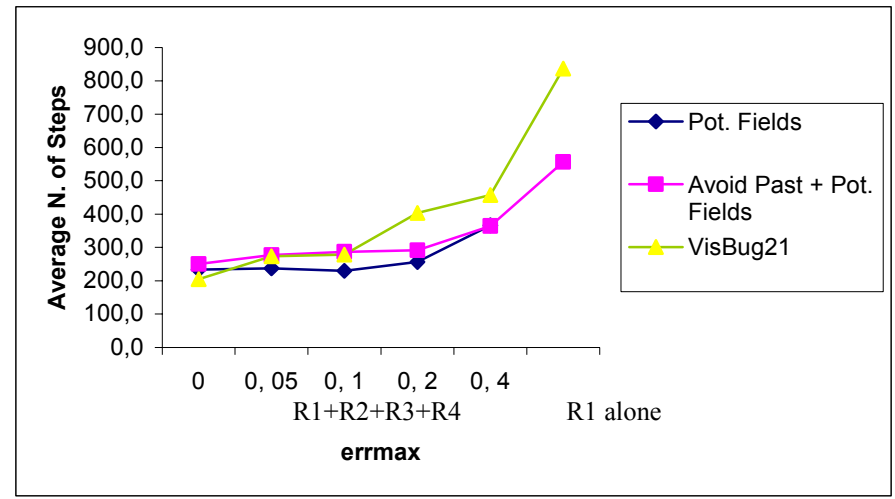

Figure 16. The results of the experiments performed in the first scenario (noise added)

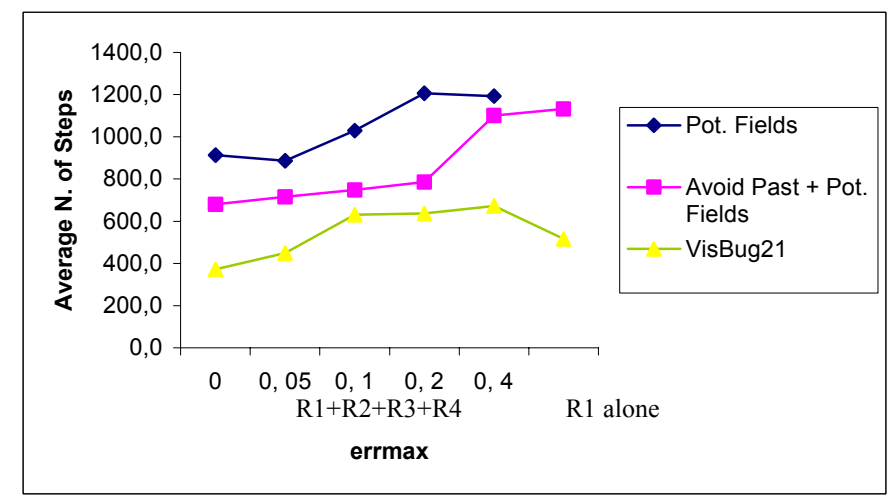

Figure 17. The results of the experiments performed in the second scenario (noise added)

\section{CONCLUSIONS.}

The work described in this paper deals with the problem of autonomous navigation and exploration in unknown indoor/outdoor scenarios (e.g. for high risk military applications). In this kind of scenario, a team of robots is deployed in order to minimize the time required for exploration and to maximize the coverage of a given area. In particular, we assume that each robot is assigned a set of goals to be achieved: goals, in our definition, can be both spatial location in the environment to be reached or particular objects to be found. The paper describes a simple (but effective) approach to the problem that allows robots to help each other in achieving their own goals when only lineof-sight communication is possible. The two strategies that have been implemented can be roughly summarized as follows: 
1. goal-sharing: a robot is attracted by teammates that 'can see' or 'have seen' its goal.

2. state-sharing: a robot 'in trouble' is attracted by teammates that are 'not in trouble'.

Different experiments have been carried out in simulated environments, showing that individual robots increase their performance when allowed to cooperate with minimal communication/memory requirements. However, the experiments carried out up to the present time take into account only a subclass of the exploration problem as it has been defined at the beginning of this paper: i.e., we considered only the case in which goals correspond with spatial location to be reached, thus ignoring the case in which robots are looking for objects whose location is unknown. Finally, notice that performance increase more if we assign some robot the role of supporting other robot in their tasks. Each support robot can help more robots at the same time and that, as we pointed out in the introduction, patrolling robots are often required for different tasks, such as watching for the presence of possible intruders or maintaining line-of-sight communication with a fixed station located at one end of the corridor for transmission to the outside world.

\section{ACKNOWLEDGMENTS}

This research has been supported in part by C.S. Draper Laboratory Inc. under contract \#DL-H-521700.

\section{REFERENCES}

[1] R.C. Arkin, 1989. "Motor Schema-Based Mobile Robot Navigation", International Journal of Robotics Research, Vol. 8, No. 4, August 1989, pp. 92-112.

[2] E. Gat, 1992. "Integrating planning and reacting in a heterogeneous asynchronous architecture for controlling real-world mobile robots", in AAAI-92, San Jose, CA, 1992, pages 809-815.

[3] J. M. Evans, 1995. "HelpMate: a Service Robot Success Story", ServiceRobot: An International Journal, Vol. 1, no.1, 1995, pp. 19-21.

[4] H. R. Everett, and D. W. Gage, 1996. "A Third Generation Security Robot", SPIE Mobile Robot and Automated Vehicle Control Systems, Boston, MA, 1996, Vol. 2903 pp. 20-21.

[5] J. Blitch, 1999. "Tactical Mobile Robots for Complex Urban Environment," Mobile Robots XIV, Boston, MA, September 1999, pp. 116-128. 
[6] Y. Koren and J. Borenstein, 1991. "Potential Field Methods and Their Inherent Limitations for Mobile Robot Navigation", Proceedings of the IEEE International Conference on Robotics and Automation, Sacramento, California, April 7-12, 1991, pp. 1398-1404.

[7] V.J. Lumelsky and T. Skewis, 1990. "Incorporating range sensing in the robot navigation function", IEEE Trans. On System Man and Cybernetics, 20(5) pp. 1058-1068, 1990

[8] T. Balch and R.C. Arkin, 1994. "Communication in Reactive Multiagent Robotic Systems", Autonomous Robots, Vol. 1, No. 1, 1994, pp. 27-52.

[9] L. Itti and C. Koch, 2000. "A saliency-based search mechanism for overt and covert shifts of visual attention", Vision Research, 40, 2000, pp. 1489-1506.

[10] B. Olshausen and C. Koch, 1995. "Selective Visual Attention," in The Handbook of Brain Theory and Neural Networks, ed. M. Arbib, MIT Press, Cambridge, MA, 1995, pp. 837-40.

[11] L.E. Parker, 1999, "Cooperative Robotics for Multi-Target Observation", Intelligent Automation and Soft Computing, special issue on Robotics Research at Oak Ridge National Laboratory, 5 (1), 1999, pp. 5-19.

[12] R. Kurazume and S. Hirose, Study on Cooperative Positioning System - Optimum Moving Strategies for CPSIII -, Proc.IEEE Int. Conf. On Robotics and Automation, Vol. 4, pp. 2896-2903, 1998.

[13] G. Dudek, M. Jenkin, E. Milios, D. Wilkes, Experiments in sensing and communication for robot convoy navigation-, Proceedings IEEE/RSJ Int. Conf. on Intelligent Robots and Systems (IROS), Pittsburgh, PA, August, 1995, Vol. 2, pp. 268-273.

[14] J. O’Rourke, 1987. Art Gallery Theorems and Algorithms, Oxford University Press, New York, NY, 1987.

[15] L.J. Guibas, J.C. Latombe, S.M. LaValle, D. Lin, and R. Motwani, "A Visibility-Based Pursuit-Evasion Problem", International Journal of Computational Geometry \& Applications, Vol. 9, Nos. 4 \& 5 (1999) 471493.

[16] R.C. Arkin, T.R. Collins, and Y. Endo, 1999 . "Tactical Mobile Robot Mission Specification and Execution", Mobile Robots XIV, Boston, MA, Sept. 1999, pp. 150-163.

[17] T. Balch, and R.C. Arkin, , 1993. "Avoiding the Past: A Simple but Effective Strategy for Reactive Navigation", Proc. 1993 IEEE International Conference on Robotics and Automation, Atlanta, GA, May 1993, Vol. 1, pp. $678-685$. 
